\title{
Numerical modeling of the solar modulation of Helium isotopes in the inner heliosphere
}

M.D. Ngobeni ${ }^{a, b, *}$, M.S. Potgieter ${ }^{c}$, O.P.M. Aslam ${ }^{a}$, D. Bisschoff ${ }^{a}$, I.I. Ramokgaba ${ }^{b}$, D.C. Ndiitwani $^{a, b}$

${ }^{a}$ Center for Space Research, North-West University, 2520, Potchefstroom, South Africa

${ }^{b}$ School of Physical and Chemical Sciences, North-West University, 2735, Mmabatho, South Africa

${ }^{c}$ Institute for Experimental and Applied Physics, Christian Albrechts University in Kiel, 24118, Kiel, Germany

E-mail: donald.ngobeni@nwu.ac.za

The observation of cosmic ray Helium isotopes at the Earth had been done precisely with the PAMELA and AMS02 space detectors and reported from July 2006 to December 2007 and from May 2011 to April 2017, respectively. These available observations span time frames that include the solar magnetic field reversal epoch. In this work, a comprehensive, threedimensional numerical modulation model for the transport of cosmic rays in the heliosphere is utilized to compute the modulation of galactic Helium isotopes from minimum activity in the previous $\mathrm{A}<0$ cycle, through solar maximum, and toward minimum activity in the current $\mathrm{A}>0$ cycle. A particular objective is to reproduce the main features of the ${ }^{3} \mathrm{He}_{2}$ to ${ }^{4} \mathrm{He}_{2}$ ratio observed at rigidities between $2.15 \mathrm{GV}$ and $15.3 \mathrm{GV}$. We find that the numerical model can reproduce the apparent single rigidity power law dependence and time dependence of this ratio above $4 \mathrm{GV}$ in good agreement with observations.

37th International Cosmic Ray Conference (ICRC 2021)

July 12 th - 23rd, 2021

Online - Berlin, Germany

*Presenter 


\section{Introduction}

The recent availability of AMS02 observations of the ${ }^{3} \mathrm{He}_{2}$ to ${ }^{4} \mathrm{He}_{2}$ ratio at rigidities between $2.15 \mathrm{GV}$ and 15.3 GV with unprecedented accuracy over a long period of time reported by [1] and complimented by PAMELA observations at energies between $\sim 0.260 \mathrm{GeV} / \mathrm{n}$ and $\sim 1.29 \mathrm{GeV} / \mathrm{n}$ reported by [2], unveiled new details whose main features warrant an investigation with our numerical model. These new measurements have revealed that ${ }^{3} \mathrm{He}_{2} /{ }^{4} \mathrm{He}_{2}$ reaches a peak around $\sim 2 \mathrm{GeV} / \mathrm{n}$ and then decreases with increasing energy. These are features previously not observed because before PAMELA and AMS02, measurements of this ratio had been characterized by a limited energy range and very large uncertainties (e.g. [3 - 5], to mention but a few). At best these measurements were obtained from balloon experiments prone to atmospheric secondary particle contamination. Therefore an extended energy dependence of this ratio could not be appropriately appreciated, let alone its dependence on rigidity which was previously completely unknown. Using AMSO2 measurements, [1] reported that ${ }^{3} \mathrm{He}_{2} /{ }^{4} \mathrm{He}_{2}$ as a function of rigidity above $4 \mathrm{GV}$ is found to be essentially time independent and can be well described by an apparent single rigidity power law, whereas below this rigidity it is found to have a significant long-term time dependence. These features about the time and rigidity dependence of this ratio are new, and an attempt is made in this paper to reproduce them with our numerical model. Previously, the lack of such precise observations of the rigidity dependence of ${ }^{3} \mathrm{He}_{2} /{ }^{4} \mathrm{He}_{2}$ over long periods of time in one place had posed a severe hindrance on numerical modeling of this ratio. Without accurate observations it has been difficult to put constraints on the very local interstellar spectra (VLIS) for both isotopes using solar modulation modeling. At best the modeling effects on ${ }^{4} \mathrm{He}_{2}$ were simply referred to as those of total Helium (e.g. [6]), entirely neglecting the contribution of ${ }^{3} \mathrm{He}_{2}$ which we consider as crucial. This neglect may seem reasonable when considering only spectra because the contribution of ${ }^{3} \mathrm{He}_{2}$ may not be directly noticeable. However, for understanding how exactly the proton to total Helium ratio (p/He), and the ratios $\mathrm{p} /{ }^{3} \mathrm{He}_{2}, \mathrm{p} /{ }^{4} \mathrm{He}_{2}$ and ${ }^{3} \mathrm{He}_{2} /{ }^{4} \mathrm{He}_{2}$ change with rigidity and time, it is crucial to separate the modulation effects on ${ }^{3} \mathrm{He}_{2}$ and ${ }^{4} \mathrm{He}_{2}$ with comprehensive modeling. This lack of detailed information about separating the modulation effects of ${ }^{3} \mathrm{He}_{2}$ and ${ }^{4} \mathrm{He}_{2}$ led [7] to conclude that there is a possibility for a fundamental astrophysics concept at play, such as that total-He may be accelerated differently than protons at Galactic cosmic ray (GCR) sources. However, [8] showed from a numerical modelling point of view, that there is no reason to believe that there are fundamental differences between these GCRs when modulated inside the heliosphere.

This paper focuses on the time and rigidity dependence of the modulation of ${ }^{3} \mathrm{He}_{2}$ and ${ }^{4} \mathrm{He}_{2}$ at the Earth from the period of minimum solar activity beginning at the end of 2006 until 2011, through the period of solar maximum activity from 2012 to just after 2014, and then for the beginning of the current period of minimum solar activity up to and including early 2017.

\section{Modulation model and numerical simulation results}

In this study a comprehensive, three-dimensional (3D) numerical model describing GCR modulation in the heliosphere is applied to the modulation of ${ }^{3} \mathrm{He}_{2}$ and ${ }^{4} \mathrm{He}_{2}$. This model is based on solving the transport equation derived by [9]: 


$$
\frac{\partial f}{\partial t}=-\left(\mathbf{V}+\left\langle\boldsymbol{v}_{D}\right\rangle\right) \cdot \nabla f+\nabla \cdot\left(\boldsymbol{K}_{s} \cdot \nabla f\right)+\frac{1}{3}(\nabla \cdot \mathbf{V}) \frac{\partial f}{\partial \ln p},
$$

where $f(\boldsymbol{r}, p, t)$ is the omnidirectional distribution function, $p$ is particle momentum, $t$ is time, and $\boldsymbol{r}$ is the 3D position vector with three coordinates $r, \theta$ and $\phi$ specified in a heliocentric spherical coordinate system where the equatorial plane is at a polar angle of $\theta=90^{\circ}$, and with $\mathbf{V}(r, \theta)=V(r, \theta) \boldsymbol{e}_{r}$ the radial SW velocity. The terms on the right-hand side represent convection, gradient and curvature drifts, diffusion, and adiabatic energy changes experienced by GCR when they enter and travel through the heliosphere up to the Earth. The details and essentials of this numerical model together with the elements of the diffusion tensor and the VLIS's of both ${ }^{3} \mathrm{He}_{2}$ and ${ }^{4} \mathrm{He}_{2}$ have been published by [8]; see also [10] for a discussion on how the drift coefficient is scaled time-dependently from 2006 till the end of 2016.

Of interest to this study are both the diffusion coefficient (DC) parallel to the average magnetic field, $K_{\|}$, and the perpendicular DC in the radial direction, $K_{\perp r}$, which are approximated by two power-laws with a smooth transition of slopes. Full details are given by [8].
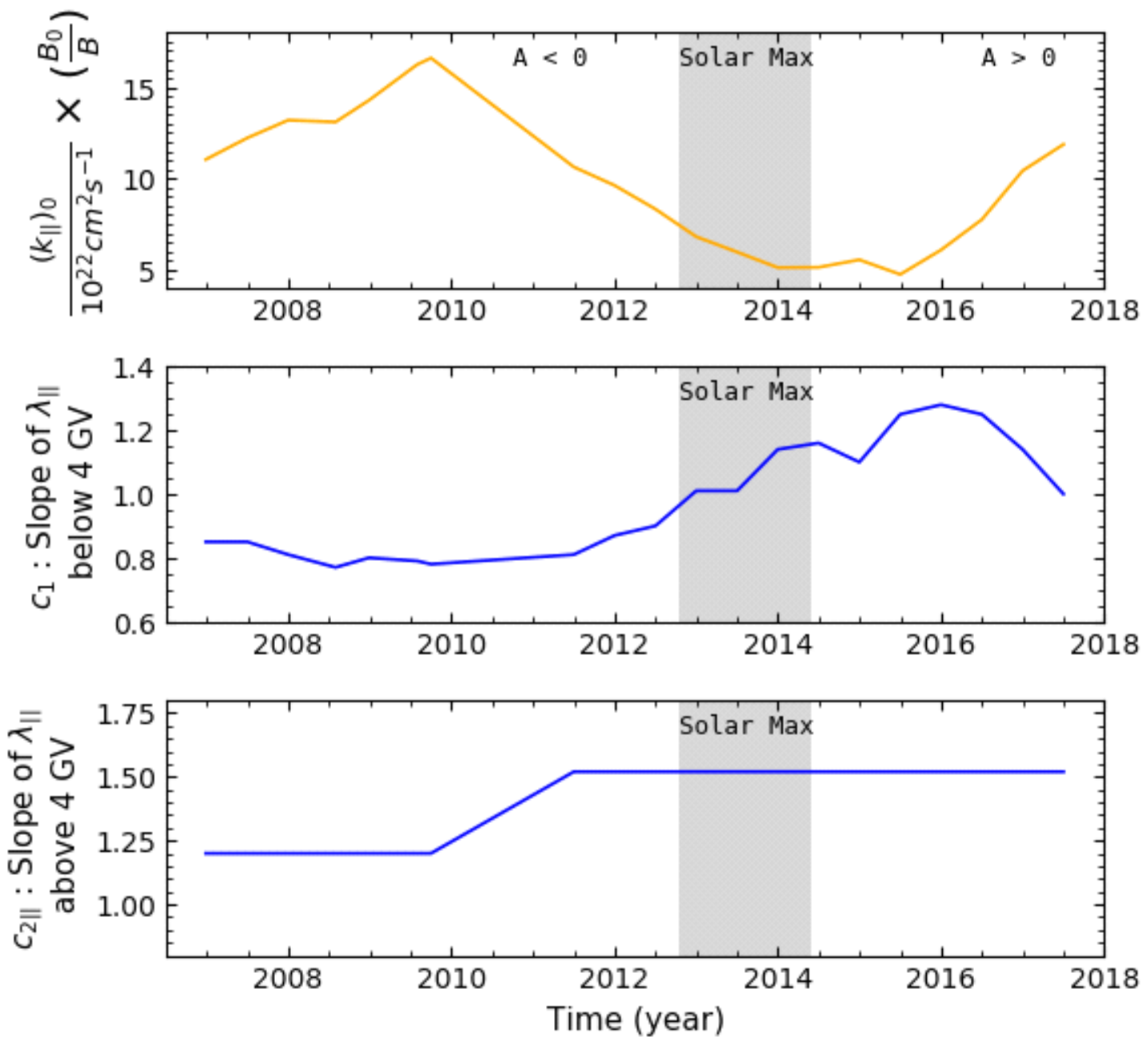

Figure 1: Time dependence of the dimensionless ratio of the scaling constant of the DCs (or MFP's) to the strength of the magnetic field at the Earth, $\left(K_{\|}\right)_{0} / B$ (top panel), the slope of the MFP's (DCs) below 4.0 $\mathrm{GV}\left(c_{1}\right.$, middle panel) and above $4.0 \mathrm{GV}\left(c_{2 \|}\right.$, bottom panel). 
Figure 1 shows the dimensionless ratio of the scaling constant of the DCs (or the equivalent mean free paths, MFPs) to the strength (magnitude) of the magnetic field, $B$, along with the rigidity dependent slopes of the MFP's (DCs) above and below 4.0 GV as a function of time, indicative of changing solar activity. The time dependence of these parameters as depicted in Figure 1 is required to reproduce the PAMELA and AMS proton and Helium observations from 2006 to 2017 (see also [11]). It is clearly noted in this figure that the rigidity slopes of the DCs below 4 GV varies modestly between 2006 and 2012, after which it increases largely with time until a maximum value is reached around 2016. This indicates that below $4 \mathrm{GV}$ the rigidity dependence of the DCs change differently before solar maximum in the $\mathrm{A}<0$ magnetic cycle than afterwards in the A $>0$ cycle. Above $4 \mathrm{GV}$, a constant value was required between 2006 and 2010, and also between 2011 and 2017 to reproduce observations.
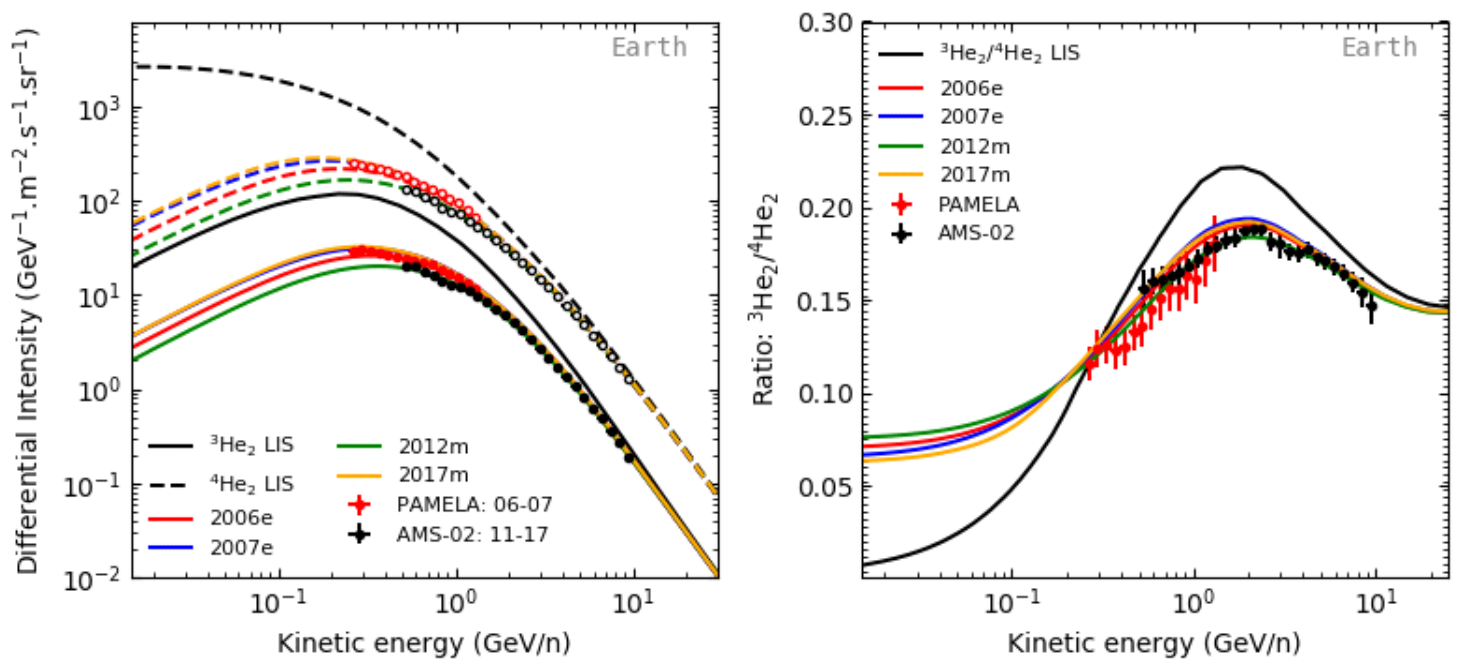

Figure 2: Left panel: VLIS for ${ }^{3} \mathrm{He}_{2}$ and ${ }^{4} \mathrm{He}_{2}$ (solid and dashed dark grey lines, respectively) specified at the HP (122 AU) are shown together with the corresponding modulated differential intensity (coloured solid and dashed lines for ${ }^{3} \mathrm{He}_{2}$ and ${ }^{4} \mathrm{He}_{2}$, respectively) computed as a function of kinetic energy per nucleon at the Earth; done for four periods corresponding to the end of the year (or half-year) indicated by different colours: 06/11/14 - 06/12/12 (2006e), 07/12/01 - 07/12/29 (2007e), 12/05/27 - 12/06/22 (2012m),17/04/13 - 17/05/09 (2017m), respectively. Right panel: Corresponding ratios of the VLIS (solid black line) and modulated spectra (solid coloured lines). Computed spectra and ratios are compared to corresponding ${ }^{3} \mathrm{He}_{2}$ and ${ }^{4} \mathrm{He}_{2}$ observations taken from [1] (AMS02, black circles and error bars) and [2] (PAMELA, red circles and error bars).

Figure 2 depicts the computed ${ }^{3} \mathrm{He}_{2}$ and ${ }^{4} \mathrm{He}_{2}$ spectra in the left panel along with the corresponding ${ }^{3} \mathrm{He}_{2} /{ }^{4} \mathrm{He}_{2}$ ratios in the right panel and overlaid on the compilation of the observed ${ }^{3} \mathrm{He}_{2}$ and ${ }^{4} \mathrm{He}_{2}$ spectra and their ratios, all shown at the Earth (1 AU in the equatorial plane with $\theta=90^{\circ}$ ). This is done with respect to the VLIS for ${ }^{3} \mathrm{He}_{2}$ and ${ }^{4} \mathrm{He}_{2}$ specified at the HP, assumed at $122 \mathrm{AU}$, for the selected periods 2006e, 2007e, 2012m, and 2017m. The PAMELA spectra of these He-isotopes are taken from [2] as observed from July 2006 to December 2007 (with A < 0 magnetic polarity during the previous solar minimum period. Whereas the AMS spectra are taken from [1] as observed between May 2011 and November 2017. The period of observations depicted in Figure 2 spans time frames that include the magnetic field reversal epoch and covers a wide energy range. This figure shows that both the PAMELA and AMS02 isotopic He spectra, together with their corresponding ratios, are reproduced quite reasonably by the numerical model; including the kinetic energy in $\mathrm{GeV} / \mathrm{n}$ where the peak in the ratio occurs, which is $\sim 2$ $\mathrm{GeV} / \mathrm{n}$. Note that in terms of rigidity, $\sim 2 \mathrm{GeV} / \mathrm{n}$ corresponds to $\sim 4.2 \mathrm{GV}$ and $\sim 5.5 \mathrm{GV}$ for ${ }^{3} \mathrm{He}_{2}$ 
and ${ }^{4} \mathrm{He}_{2}$, respectively. Therefore, it is important to illustrate how the spectra and ratio of both isotopes evolve with time at the same rigidity; which is what we focus on next. We consider the good compatibility with observations shown in Figure 2 as to indicate that the shape of the LIS's of ${ }^{3} \mathrm{He}_{2}$ and ${ }^{4} \mathrm{He}_{2}$ obtained from the GALPROP model as described by [12] but modestly adjusted according to AMS02 observations for ${ }^{3} \mathrm{He}_{2} /{ }^{4} \mathrm{He}_{2}$ as shown in Figure 4, generally are reasonable to use in a detail study of modeling the modulation of these isotopes in the inner heliosphere.
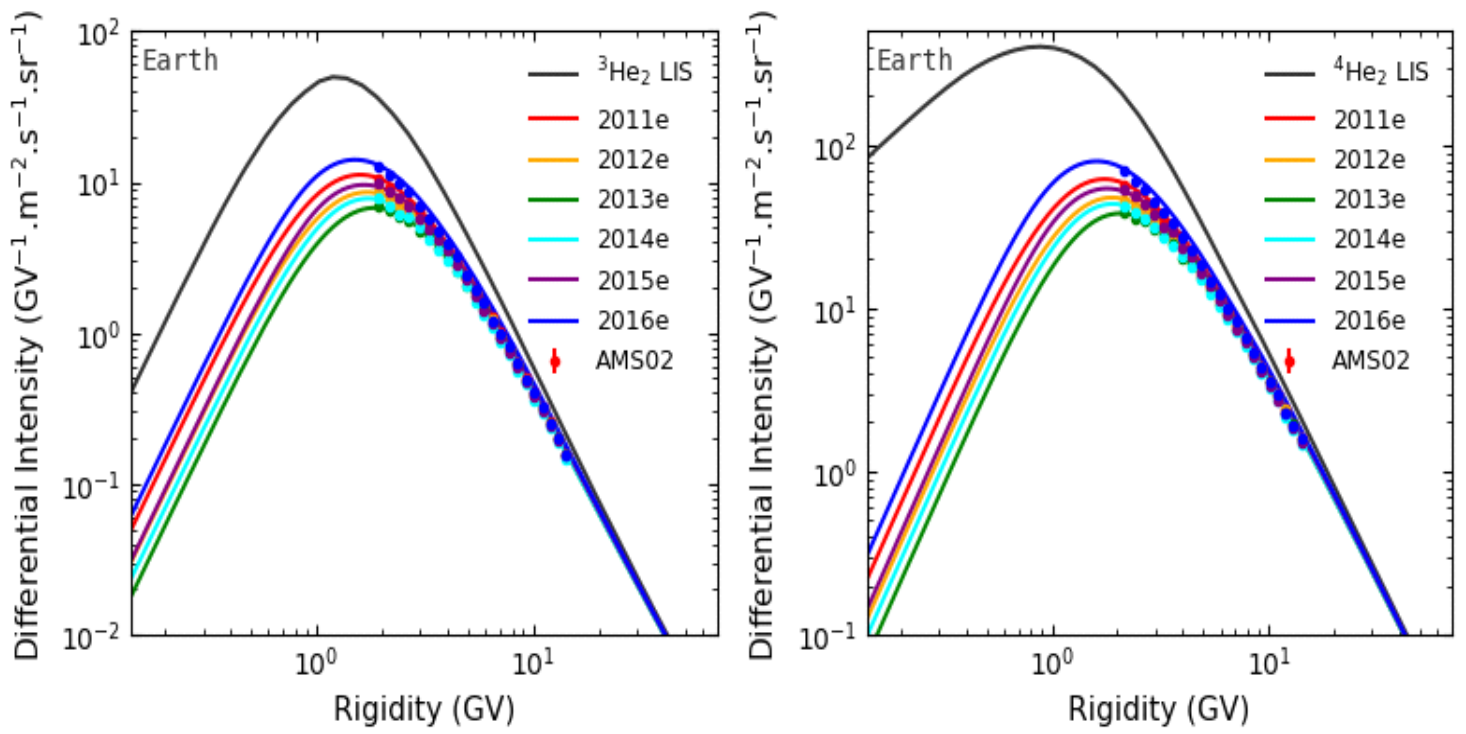

Figure 3: Left panel: VLIS is shown for ${ }^{3} \mathrm{He}_{2}$ (dark grey lines), specified at the HP (122 AU), together with the modulated differential intensity (coloured solid lines) computed as a function of rigidity for the time period including Bartel rotations (BR) at the end of the year indicated by different colours: 2433 (11/11/20 - 11/12/16; 2011; 2011e), 2447 (12/12/02 - 12/12/28; 2012e), 2460 (13/11/18 - 13/12/14; 2013e), 2474 (14/12/01 - 14/12/27; 2014e), 2487 (15/11/17 - 15/12/13; 2015e) and 2501 (16/11/29 $16 / 12 / 25$; 2016e), respectively. These computed spectra are compared to AMS observations of ${ }^{3} \mathrm{He}_{2}$ (coloured filled circles). Right panel: Similar to the left panel but for the VLIS of ${ }^{4} \mathrm{He}_{2}$ (dark grey line) and the corresponding modulated spectra (coloured solid lines), compared with AMS observations of ${ }^{4} \mathrm{He}_{2}$ (coloured filled circles). Both ${ }^{3} \mathrm{He}_{2}$ and ${ }^{4} \mathrm{He}_{2}$ AMS observations are reported by [1]

Figure 3 depicts the computed ${ }^{3} \mathrm{He}_{2}$ (left panel) and ${ }^{4} \mathrm{He}_{2}$ (right panel) modulated spectra with respect to the respective VLIS as a function of rigidity, in comparison with the corresponding observed AMS02 spectra at the Earth. The computed results and observations are shown from 2011e to 2016e. The essence of this figure is to show that the ${ }^{3} \mathrm{He}_{2}$ and ${ }^{4} \mathrm{He}_{2}$ spectra from AMS02 are well reproduced by our numerical model. These computations are obtained using the same set of modulation parameters, DCs and drift coefficient that reproduce the AMS02 proton modulation between 2011 and 2017 (see also [13]; [11]).

Together, Figures 1 and 3 aims to illustrate that only changing $B$ and the tilt angle from 2006 to 2017 with time cannot reproduce all the observed spectra, therefore, we find that it is crucial to change the values and the rigidity slopes of the DCs as shown in Figure 1, together with the drift coefficient as reported by [10]. Next, we illustrate the time dependence in the modulation of ${ }^{3} \mathrm{He}_{2}$ to that of ${ }^{4} \mathrm{He}_{2}$ at rigidities between $2.15 \mathrm{GV}$ and $2.40 \mathrm{GV}$, which spans a typical rigidity bin used in AMSO2 reports. At these rigidities, the measured ${ }^{3} \mathrm{He}_{2} /{ }^{4} \mathrm{He}_{2}$ has shown significant declines in response to the declining solar activity levels ([1]). 


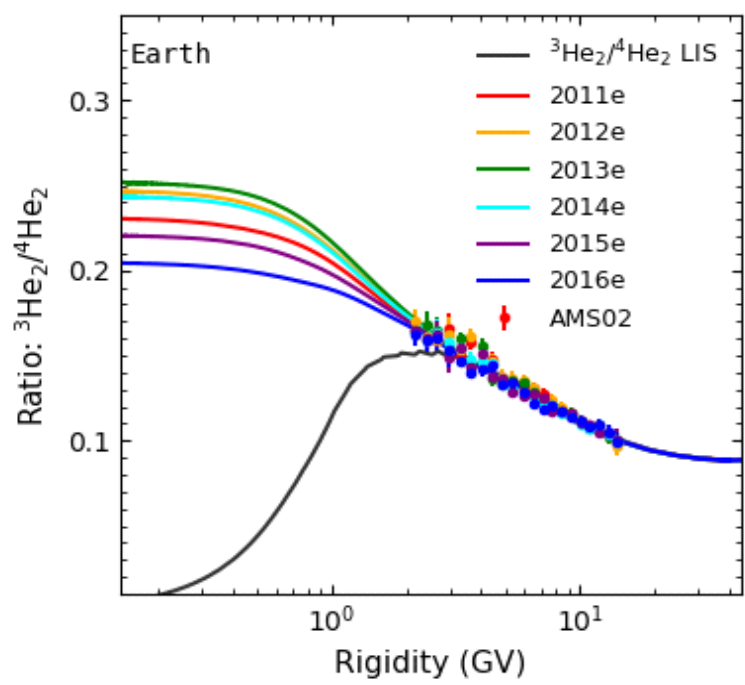

Figure 4: The computed ${ }^{3} \mathrm{He}_{2} /{ }^{4} \mathrm{He}_{2}$ ratios (coloured solid lines) as a function of rigidity for six BR periods between 2011e and 2016e are compared with AMS02 observations from [1] taken at the same periods. As a reference the corresponding ratio of the VLIS for these two isotopes is given by dark grey line.
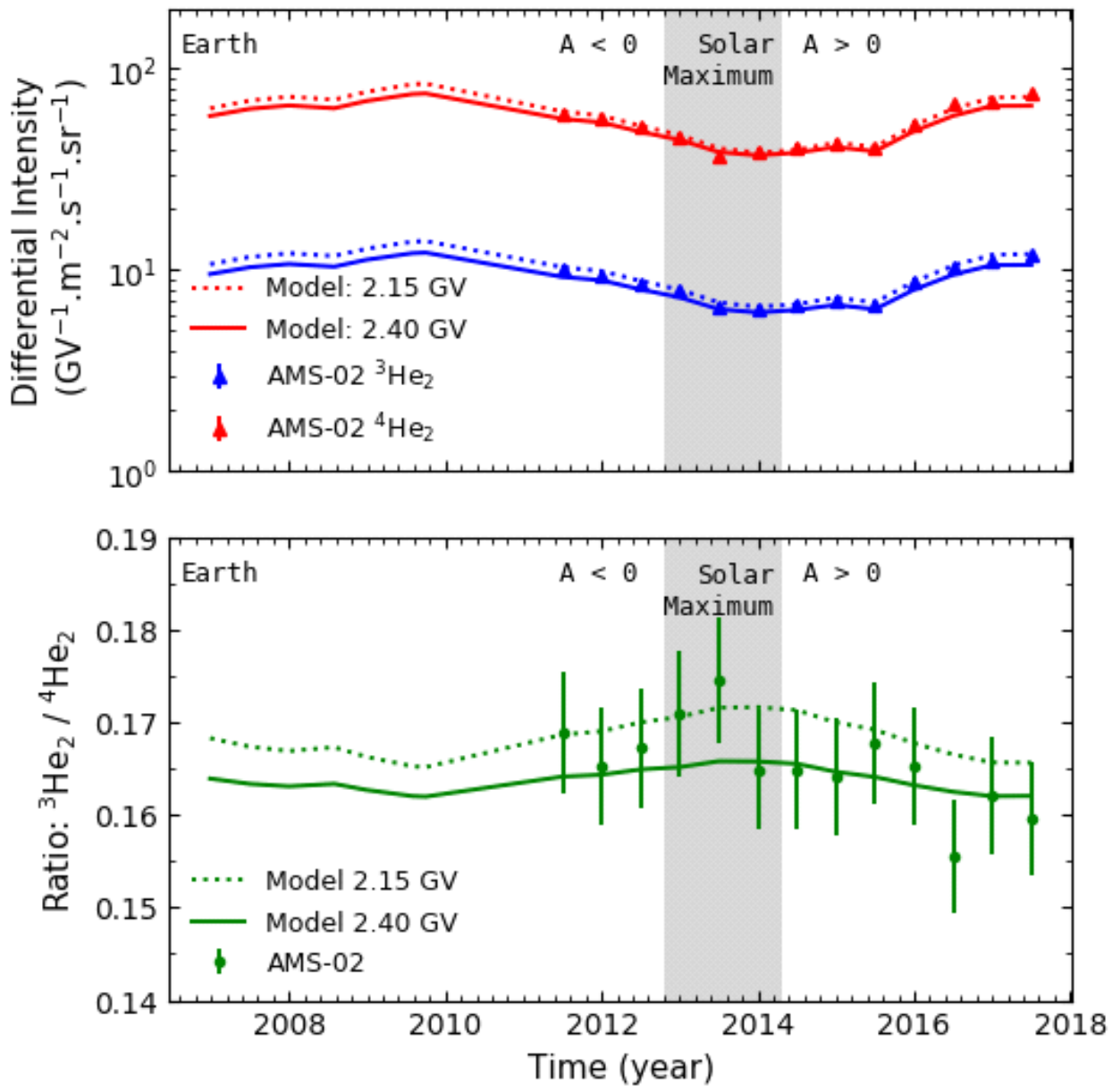

Figure 5: Top Panel: Computed ${ }^{3} \mathrm{He}_{2}$ (blue lines) and ${ }^{4} \mathrm{He}_{2}$ (red lines) at a rigidity of $2.15 \mathrm{GV}$ (dotted lines) and 2.40 GV (solid lines), respectively, in comparison with AMS02 intensities reported for the rigidity range $2.15-2.40 \mathrm{GV}$ as a function of time, also indicating the $\mathrm{A}<0$ and $\mathrm{A}>0$ epochs before and afterward the period of maximum solar activity. Bottom panel shows the corresponding computed ${ }^{3} \mathrm{He}_{2} /{ }^{4} \mathrm{He}_{2}$ together with AMS02 observations reported by [1]. 
The computed ratios, ${ }^{3} \mathrm{He}_{2} /{ }^{4} \mathrm{He}_{2}$, from 2011e to 2016e are shown at the Earth as a function of rigidity with respect to their corresponding VLIS's in Figure 4 and in comparison with AMS02 observations. It follows that the numerical computations can reproduce convincingly the apparent single rigidity power law dependence, as well as the time independence of this ratio, above $4 \mathrm{GV}$. Whereas below $\sim 2 \mathrm{GV}$, where there are not any AMS data, the computed ratios significantly decrease with decreasing rigidity from its maximum value reached during solar maximum conditions as represented by 2013e. We note that at rigidities below 2 GV, PAMELA observations with a lower rigidity thresh-hold are well suited to study the large time dependence in ${ }^{3} \mathrm{He}_{2} /{ }^{4} \mathrm{He}_{2}$ as computed in Figure 4. However, AMS measurements with good statistics make it possible to study precisely the time variation of ${ }^{3} \mathrm{He}_{2} /{ }^{4} \mathrm{He}_{2}$ through maximum solar activity, in essence how it behaves before and after solar maximum.

The top panel of Figure 5 displays the quantitative and qualitative time-dependent modulation of the computed ${ }^{3} \mathrm{He}_{2}$ and ${ }^{4} \mathrm{He}_{2}$ spectra at rigidities of $2.15 \mathrm{GV}$ and $2.40 \mathrm{GV}$, compared to the observed AMS intensities with their systematic errors in the $2.15 \mathrm{GV}-2.40 \mathrm{GV}$ rigidity bin. The corresponding ${ }^{3} \mathrm{He}_{2} /{ }^{4} \mathrm{He}_{2}$ are shown in the bottom panel. It follows for the displayed time scale that the measured ${ }^{3} \mathrm{He}_{2}$ and ${ }^{4} \mathrm{He}_{2}$ fluxes show nearly identical time dependence and relative variations in amplitude. After 2015, both ${ }^{3} \mathrm{He}_{2}$ and ${ }^{4} \mathrm{He}_{2}$ intensities have started recovering, in response to declining solar activity levels, and during this period the computed ${ }^{3} \mathrm{He}_{2} /{ }^{4} \mathrm{He}_{2}$ decreases consistent with the AMS02 observations, more so for the lower rigidity. This is mainly caused by the rigidity slopes of the DCs, as shown in Figure 1, that change differently before solar maximum than afterwards, and that this influences somewhat differently the modulation of GCRs with different VLIS's and A/Z values. Overall, there is good compatibility between the time profiles of the computed and observed ratios. From Figure 5, it is further noted that the computed values of ${ }^{3} \mathrm{He}_{2} /{ }^{4} \mathrm{He}_{2}$ for the period of decreasing (increasing) ratio coincide with the increasing (decreasing) intensities of both isotopes, which may well be a general modulation pattern.

\section{Summary and conclusion}

Our 3D drift model [8] is applied to the modulation of the two Helium isotopes, ${ }^{3} \mathrm{He}_{2}$ and ${ }^{4} \mathrm{He}_{2}$. This requires that, first, the VLIS's of both ${ }^{3} \mathrm{He}_{2}$ and ${ }^{4} \mathrm{He}_{2}$ must be obtained by applying GALPROP models as described by [12], refined and compared to the existing set of accurate observations as a function of kinetic energy per nucleon, shown in Figure 2. This obtained compatibility indicates that in general these VLIS's can be utilized as input spectra for the detail study of modelling He-isotopes in the inner heliosphere. As mentioned, the observed features of the time and rigidity dependence of ${ }^{3} \mathrm{He}_{2} /{ }^{4} \mathrm{He}_{2}$ are new. The results presented here confirm that our numerical model can reproduce satisfactorily modulation effects for ${ }^{3} \mathrm{He}_{2}$ and ${ }^{4} \mathrm{He}_{2}$, both in terms of their spectra and the corresponding ratio. Above $4.0 \mathrm{GV},{ }^{3} \mathrm{He}_{2} /{ }^{4} \mathrm{He}_{2}$ was found to have an almost single rigidity power law and it seems independent of the assumed modulation conditions, while below 4.0 GV, the computed ${ }^{3} \mathrm{He}_{2} /{ }^{4} \mathrm{He}_{2}$, from early 2014 at the end of the solar maximum activity period, decreases as a function of time consistently in good agreement with the AMS observations. To reproduce these observations, we find that in addition to scaling down (up) the values of the diffusion and drift coefficients towards (after) the solar maximum, the rigidity slopes of the parallel and perpendicular DCs below 4 GV should change differently before solar maximum than afterwards as illustrated in Figure 1. The time dependence in the 
rigidity slopes of the DCs influences somewhat differently the modulation of GCR particles with different VLIS's and A/Z's.

\section{Acknowldgement}

MDN thanks the SA National Research Foundation (NRF) for partial financial support under Joint Science and Technology Research Collaboration (Grant no:118915) and BAAP (Grant no: 120642). DB and OPMA acknowledge the financial support from the NWU post-doctoral programme.

\section{References}

[1] M. Aguilar et al., Phys.Rev. Lett. 123, 181102 (2019)

[2] O. Adriani et al., Astrophys. J, 818, 68 (2016)

[3] O. Reimer et al., Astrophys. J. 496, 490, (1998)

[4] J.Z. Wang et al., Astrophys. J. 564, 244, (2002)

[5] Z.D. Myers et al., ICRC 4, 1805 (2003)

[6] Z.N. Shen et al., Astrophys. J, 887, 132 (2019)

[7] N. Tomassetti, Astrophys. Lett, 815, L1 (2015)

[8] M.D. Ngobeni et al., Astrophys \& Space Sci. 365, 182 (2020)

[9] E.N. Parker, Planet. Space Sci, 13, 9 (1965)

[10] O.P.M. Aslam et al. Astrophys. J, 909, 215 (2021)

[11] M.D. Ngobeni et al., Proc. Sci (ICRC 2021)

[12] D. Bisschoff et al., Astrophys. J, 878, 59 (2019)

[13] O.P.M. Aslam et al. Proc. Sci (ICRC 2019), 1054 